\title{
On the periodicity of a max-type rational difference equation
}

\author{
Changyou Wang ${ }^{\mathrm{a}, \mathrm{b}, *}$, Xiaotong Jing ${ }^{\mathrm{b}}$, Xiaohong $\mathrm{Hu}^{\mathrm{b}}$, Rui Li $\mathrm{Li}^{\mathrm{c}, *}$ \\ a School of Applied Mathematics, Chengdu University of Information Technology, Chengdu, Sichuan 610225, P. R. China. \\ ${ }^{b}$ College of Science, Chongqing University of Posts and Telecommunications, Chongqing 400065, P. R. China. \\ ${ }^{c}$ College of Automation, Chongqing University of Posts and Telecommunications, Chongqing 400065, P. R. China.
}

Communicated by K. Q. Lan

\begin{abstract}
This paper shows that every well-defined solution of the following max-type difference equation

$$
x_{n+1}=\max \left\{\frac{A}{x_{n}}, \frac{A}{x_{n-1}}, x_{n-2}\right\}, \quad n \in N_{0},
$$

where $A \in R$ and the initial conditions $x_{-2}, x_{-1}, x_{0}$ are arbitrary non-zero real numbers is eventually periodic with period three by using new iteration method for the more general nonlinear difference equations and inequality skills as well as the mathematical induction. Our main results considerably improve results appearing in the literature. (C) 2017 All rights reserved.
\end{abstract}

Keywords: Max-type, difference equation, positive solution, periodic solution. 2010 MSC: 39A10.

\section{Introduction}

Although difference equations which appear naturally as discrete analogues in the numerical solutions of differential and delay differential equations have been predominantly studied so far (see, for example, $[11,16,21]$ and the references therein), the study of nonlinear difference equations which are not discrete analogues of differential equations has been also of a great interest recently (see, for example, $[7,9,13]$ and the references therein). Recently, so called, max-type difference equations have attracted some attention (see, for example, $[6,17,20]$ and the references therein) because the max operator have great importance in automatic control models (see, $[15,19])$ and have wide applications in biology (see, [8]), ecology (see, [12, $18]$ ), and physics (see, $[4,10])$. However, the maxima operator is not a smooth function in $n$-dimensional real vector space so that the techniques which use derivatives could be of almost no use, so the study of max-type systems of difference equations become more difficult. In 2002, Mishev et al. [14] considered the positive solutions of the following difference equation

$$
x_{n+1}=\max \left\{\frac{A}{x_{n}}, \frac{B}{x_{n-2}}\right\}, n=0,1,2, \cdots,
$$

\footnotetext{
*Corresponding authors

Email addresses: wangcy@cqupt.edu.cn (Changyou Wang), liruimath@qq.com (Rui Li)
} 
where $A, B$ are any positive coefficients and the initial values $x_{-2}, x_{-1}, x_{0}$ are any positive numbers, and proved that every positive solution is eventually periodic. In 2009, Elsayed and Stević [3] showed that every well-defined solution of the difference equation

$$
x_{n+1}=\max \left\{\frac{A}{x_{n}}, x_{n-2}\right\}, n \in N_{0},
$$

where $A \in R$ is eventually periodic with period three. In [25], Xiao et al. showed that every well-defined solution of the following difference equation

$$
x_{n+1}=\max \left\{\frac{\beta}{x_{n}}, x_{n-1}\right\}, n \in N_{0},
$$

where the initial conditions $x_{-1}, x_{0}$ are arbitrary non-zero real numbers and $\beta \in R$, is eventually periodic with period two. Sun et al. [24] studied the global behavior of the following max-type difference equation

$$
x_{n}=\max \left\{\frac{1}{x_{n-m}}, \frac{A_{n}}{x_{n-r}}\right\}, n=0,1,2, \cdots,
$$

where $\left\{A_{n}\right\}_{n} \geqslant 0$ is a sequence of positive numbers with $A_{n} \in(0,1)$ for every $n \geqslant 0$ and $\sup A_{n}<1$, and $m, r \in\{1,2,3, \cdots\}$, and the initial values are positive real number.

Motivated by this line of investigations, our aim here is to show that the solution of the following max-type difference equation

$$
x_{n+1}=\max \left\{\frac{A}{x_{n}}, \frac{A}{x_{n-1}}, x_{n-2}\right\}, n \in N_{0},
$$

where the initial conditions $x_{-2}, x_{-1}, x_{0}$ are arbitrary non-zero real numbers and $A \in R$ is eventually periodic with period three. For closely related papers in this research area, see, for example, $[1,2,5,22,23]$ and the references therein.

Remark 1.1. Note that if $A=0$, then the Eq. (1.1) becomes $x_{n+1}=x_{n-2}$, from which it follows that every solution is periodic with period three. Therefore, in the sequel we will consider the case $A \neq 0$.

\section{Main results}

\subsection{An auxiliary result}

In this section we will prove a simple auxiliary result which will be used many times in the rest of the paper.

Lemma 2.1. Assume that $\left\{x_{n}\right\}_{n=-2}^{\infty}$ is a solution of $E q$. (1.1) and there is $k_{0} \in \mathrm{N}_{0} \cup\{-2,-1\}$ such that

$$
\mathrm{x}_{\mathrm{k}_{0}}=\mathrm{x}_{\mathrm{k}_{0}+3}, \quad \mathrm{x}_{\mathrm{k}_{0}+1}=\mathrm{x}_{\mathrm{k}_{0}+4}, \quad \mathrm{x}_{\mathrm{k}_{0}+2}=\mathrm{x}_{\mathrm{k}_{0}+5} .
$$

Then this solution is eventually periodic with period three.

Proof. We prove that

$$
x_{k_{0}}=x_{k_{0}+3 m}, \quad x_{k_{0}+1}=x_{k_{0}+1+3 m}, \quad x_{k_{0}+2}=x_{k_{0}+2+3 m}
$$

for every $m \in N$, from which the lemma follows.

We use the method of induction. For $m=1,(2.2)$ becomes (2.1). Assume that (2.2) holds for $1 \leqslant m \leqslant$ $\mathrm{m}_{0}$. From this and by using (1.1) and (2.1) as well as iterative method, one can obtain

$$
\begin{gathered}
x_{k_{0}+3\left(m_{0}+1\right)}=\max \left\{\frac{A}{x_{k_{0}+3 m_{0}+2}}, \frac{A}{x_{k_{0}+3 m_{0}+1}}, x_{k_{0}+3 m_{0}}\right\}=\max \left\{\frac{A}{x_{k_{0}+2}}, \frac{A}{x_{k_{0}+1}}, x_{k_{0}}\right\}=x_{k_{0}+3}=x_{k_{0}}, \\
x_{k_{0}+1+3\left(m_{0}+1\right)}=\max \left\{\frac{A}{x_{k_{0}+3 m_{0}+3}}, \frac{A}{x_{k_{0}+3 m_{0}+2}}, x_{k_{0}+3 m_{0}+1}\right\}=\max \left\{\frac{A}{x_{k_{0}+3}}, \frac{A}{x_{k_{0}+2}}, x_{k_{0}+1}\right\}=x_{k_{0}+4}=x_{k_{0}+1}, \\
x_{k_{0}+2+3\left(m_{0}+1\right)}=\max \left\{\frac{A}{x_{k_{0}+3 m_{0}+4}}, \frac{A}{x_{k_{0}+3 m_{0}+3}}, x_{k_{0}+3 m_{0}+2}\right\}=\max \left\{\frac{A}{x_{k_{0}+4}}, \frac{A}{x_{k_{0}+3}}, x_{k_{0}+2}\right\}=x_{k_{0}+5}=x_{k_{0}+2},
\end{gathered}
$$

the proof is completed. 


\subsection{The case $\mathrm{A}>0$}

In this section, we will consider the case $A>0$ of Eq. (1.1). Firstly we prove another auxiliary result which is incorporated in the following lemma, and then we formulate and prove the main results in this section.

Lemma 2.2. Assume that $A>0$. Then every solution of Eq. (1.1) is eventually positive if initial conditions satisfy one of the following conditions

(i) $x_{-2}, x_{-1}, x_{0}>0$;

(ii) $x_{-1}, x_{0}>0, x_{-2}<0$;

(iii) $x_{-2}, x_{0}>0, x_{-1}<0$;

(iv) $x_{-2}, x_{-1}>0, x_{0}<0$;

(v) $x_{0}>0, x_{-2}, x_{-1}<0$;

(vi) $x_{-2}>0, x_{-1}, x_{0}<0$;

(vii) $x_{-1}>0, x_{-2}, x_{0}<0$.

Proof. If $x_{0}>0$ or $x_{-1}>0$ or $x_{-2}>0$, then one has

$$
x_{1}=\max \left\{\frac{A}{x_{0}}, \frac{A}{x_{-1}}, x_{-2}\right\}>0 .
$$

From this, (1.1) and by induction and iterative method it follows that $x_{n}>0$ for every $n \in N_{0}$.

Theorem 2.3. Assume that A $>0$. Then every solution of Eq. (1.1) with positive initial conditions is eventually periodic with period three.

Proof. From Eq. (1.1), we can obtain that

$$
x_{1}=\max \left\{\frac{A}{x_{0}}, \frac{A}{x_{-1}}, x_{-2}\right\} .
$$

There are three cases to be considered.

Case 1. Assume that $\frac{A}{x_{0}} \geqslant \frac{A}{x_{-1}}$ and $\frac{A}{x_{0}} \geqslant x_{-2}$, then $x_{1}=\frac{A}{x_{0}}, x_{-1} \geqslant x_{0}, \frac{A}{x_{-2}} \geqslant x_{0}$, thus we have

$$
x_{2}=\max \left\{\frac{A}{x_{1}}, \frac{A}{x_{0}}, x_{-1}\right\}=\max \left\{x_{0}, \frac{A}{x_{0}}, x_{-1}\right\} .
$$

$\left(a_{1}\right)$ Assume that $\frac{A}{x_{0}} \geqslant x_{-1} \geqslant x_{0}$, then $x_{2}=\frac{A}{x_{0}}$, and from the iterative method we have

$$
\begin{aligned}
& x_{3}=\max \left\{\frac{A}{x_{2}}, \frac{A}{x_{1}}, x_{0}\right\}=\max \left\{x_{0}, x_{0}, x_{0}\right\}=x_{0}, \\
& x_{4}=\max \left\{\frac{A}{x_{3}}, \frac{A}{x_{2}}, x_{1}\right\}=\max \left\{\frac{A}{x_{0}}, x_{0}, \frac{A}{x_{0}}\right\}=\frac{A}{x_{0}}, \\
& x_{5}=\max \left\{\frac{A}{x_{4}}, \frac{A}{x_{3}}, x_{2}\right\}=\max \left\{x_{0}, \frac{A}{x_{0}}, \frac{A}{x_{0}}\right\}=\frac{A}{x_{0}} .
\end{aligned}
$$

Hence $x_{3}=x_{0}, x_{4}=x_{1}, x_{5}=x_{2}$. From this and by Lemma 2.1 we have that the solution is eventually periodic with period three. Moreover, from the induction and iterative method we have

$$
x_{3 n}=x_{0}, x_{3 n+1}=\frac{A}{x_{0}}, x_{3 n+2}=\frac{A}{x_{0}}, \quad n \in N_{0} .
$$

In the meantime, we can find the solution of Eq. (1.1) has the following form

$$
\left\{x_{-2}, x_{-1}, x_{0}, \frac{A}{x_{0}}, \frac{A}{x_{0}}, x_{0}, \frac{A}{x_{0}}, \frac{A}{x_{0}}, \cdots\right\}
$$


$\left(a_{2}\right)$ Assume that $x_{-1} \geqslant \frac{A}{x_{0}} \geqslant x_{0}$, then $x_{2}=x_{-1}, x_{0} \geqslant \frac{A}{x_{-1}}$. From the induction and iterative method we have

$$
\begin{aligned}
& x_{3}=\max \left\{\frac{A}{x_{2}}, \frac{A}{x_{1}}, x_{0}\right\}=\max \left\{\frac{A}{x_{-1}}, x_{0}, x_{0}\right\}=x_{0}, \\
& x_{4}=\max \left\{\frac{A}{x_{3}}, \frac{A}{x_{2}}, x_{1}\right\}=\max \left\{\frac{A}{x_{0}}, \frac{A}{x_{-1}}, \frac{A}{x_{0}}\right\}=\frac{A}{x_{0}}, \\
& x_{5}=\max \left\{\frac{A}{x_{4}}, \frac{A}{x_{3}}, x_{2}\right\}=\max \left\{x_{0}, \frac{A}{x_{0}}, x_{-1}\right\}=x_{-1} .
\end{aligned}
$$

Hence $x_{3}=x_{0}, x_{4}=x_{1}, x_{5}=x_{2}$. From this and by Lemma 2.1 we have that the solution is eventually periodic with period three. Moreover, we have

$$
x_{3 n}=x_{0}, x_{3 n+1}=\frac{A}{x_{0}}, x_{3 n+2}=x_{-1}, \quad n \in N_{0},
$$

and we can find the solution of Eq. (1.1) has the following form

$$
\left\{x_{-2}, x_{-1}, x_{0}, \frac{A}{x_{0}}, x_{-1}, x_{0}, \frac{A}{x_{0}}, x_{-1}, \cdots\right\} .
$$

$\left(a_{3}\right)$ Assume that $x_{-1} \geqslant x_{0} \geqslant \frac{A}{x_{0}}$, then $x_{2}=x_{-1}, x_{0} \geqslant \frac{A}{x_{-1}}$ and we have

$$
\begin{aligned}
& x_{3}=\max \left\{\frac{A}{x_{2}}, \frac{A}{x_{1}}, x_{0}\right\}=\max \left\{\frac{A}{x_{-1}}, x_{0}, x_{0}\right\}=x_{0}, \\
& x_{4}=\max \left\{\frac{A}{x_{3}}, \frac{A}{x_{2}}, x_{1}\right\}=\max \left\{\frac{A}{x_{0}}, \frac{A}{x_{-1}}, \frac{A}{x_{0}}\right\}=\frac{A}{x_{0}}, \\
& x_{5}=\max \left\{\frac{A}{x_{4}}, \frac{A}{x_{3}}, x_{2}\right\}=\max \left\{x_{0}, \frac{A}{x_{0}}, x_{-1}\right\}=x_{-1} .
\end{aligned}
$$

Hence $x_{3}=x_{0}, x_{4}=x_{1}, x_{5}=x_{2}$. From this and by Lemma 2.1 we have that the solution is eventually periodic with period three. Moreover, we have

$$
x_{3 n}=x_{0}, x_{3 n+1}=\frac{A}{x_{0}}, x_{3 n+2}=x_{-1}, \quad n \in N_{0},
$$

and we can find the solution of Eq. (1.1) has the following form

$$
\left\{x_{-2}, x_{-1}, x_{0}, \frac{A}{x_{0}}, x_{-1}, x_{0}, \frac{A}{x_{0}}, x_{-1}, \cdots\right\}
$$

Case 2. Assume that $x_{-2} \geqslant \frac{A}{x_{0}}$ and $x_{-2} \geqslant \frac{A}{x_{-1}}$, then $x_{1}=x_{-2}, x_{-1} \geqslant \frac{A}{x_{-2}}, x_{0} \geqslant \frac{A}{x_{-2}}$, and we have

$$
x_{2}=\max \left\{\frac{A}{x_{1}}, \frac{A}{x_{0}}, x_{-1}\right\}=\max \left\{\frac{A}{x_{-2}}, \frac{A}{x_{0}}, x_{-1}\right\} \text {. }
$$

$\left(b_{1}\right)$ Assume that $\frac{A}{x_{0}} \geqslant x_{-1} \geqslant \frac{A}{x_{-2}}$, then $x_{2}=\frac{A}{x_{0}}, x_{-2} \geqslant x_{0}$, and we have

$$
\begin{aligned}
& x_{3}=\max \left\{\frac{A}{x_{2}}, \frac{A}{x_{1}}, x_{0}\right\}=\max \left\{x_{0}, \frac{A}{x_{-2}}, x_{0}\right\}=x_{0}, \\
& x_{4}=\max \left\{\frac{A}{x_{3}}, \frac{A}{x_{2}}, x_{1}\right\}=\max \left\{\frac{A}{x_{0}}, x_{0}, x_{-2}\right\}=x_{-2}, \\
& x_{5}=\max \left\{\frac{A}{x_{4}}, \frac{A}{x_{3}}, x_{2}\right\}=\max \left\{\frac{A}{x_{-2}}, \frac{A}{x_{0}}, \frac{A}{x_{0}}\right\}=\frac{A}{x_{0}} .
\end{aligned}
$$

Hence $x_{3}=x_{0}, x_{4}=x_{1}, x_{5}=x_{2}$. From this and by Lemma 2.1 we have that the solution is eventually 
periodic with period three. Moreover, we have

$$
x_{3 n}=x_{0}, x_{3 n+1}=x_{-2}, x_{3 n+2}=\frac{A}{x_{0}}, \quad n \in N_{0},
$$

and we can find the solution of Eq. (1.1) has the following form

$$
\left\{x_{-2}, x_{-1}, x_{0}, x_{-2}, \frac{A}{x_{0}}, x_{0}, x_{-2}, \frac{A}{x_{0}}, \cdots\right\} .
$$

$\left(b_{2}\right)$ Assume that $x_{-1} \geqslant \frac{A}{x_{0}} \geqslant \frac{A}{x_{-2}}$, then $x_{2}=x_{-1}, x_{0} \geqslant \frac{A}{x_{-1}}$, and we have

$$
\begin{aligned}
& x_{3}=\max \left\{\frac{A}{x_{2}}, \frac{A}{x_{1}}, x_{0}\right\}=\max \left\{\frac{A}{x_{-1}}, \frac{A}{x_{-2}}, x_{0}\right\}=x_{0}, \\
& x_{4}=\max \left\{\frac{A}{x_{3}}, \frac{A}{x_{2}}, x_{1}\right\}=\max \left\{\frac{A}{x_{0}}, \frac{A}{x_{-1}}, x_{-2}\right\}=x_{-2} \\
& x_{5}=\max \left\{\frac{A}{x_{4}}, \frac{A}{x_{3}}, x_{2}\right\}=\max \left\{\frac{A}{x_{-2}}, \frac{A}{x_{0}}, x_{-1}\right\}=x_{-1} .
\end{aligned}
$$

Hence $x_{3}=x_{0}, x_{4}=x_{1}, x_{5}=x_{2}$. From this and by Lemma 2.1 we have that the solution is eventually periodic with period three. Moreover, we have

$$
x_{3 n}=x_{0}, x_{3 n+1}=x_{-2}, x_{3 n+2}=x_{-1}, \quad n \in N_{0},
$$

and we can find the solution of Eq. (1.1) has the following form

$$
\left\{x_{-2}, x_{-1}, x_{0}, x_{-2}, x_{-1}, x_{0}, x_{-2}, x_{-1}, \cdots\right\} \text {. }
$$

(b3) Assume that $x_{-1} \geqslant \frac{A}{x_{-2}} \geqslant \frac{A}{x_{0}}$, then $x_{2}=x_{-1}, x_{0} \geqslant \frac{A}{x_{-1}}$, and we have

$$
\begin{aligned}
& x_{3}=\max \left\{\frac{A}{x_{2}}, \frac{A}{x_{1}}, x_{0}\right\}=\max \left\{\frac{A}{x_{-1}}, \frac{A}{x_{-2}}, x_{0}\right\}=x_{0}, \\
& x_{4}=\max \left\{\frac{A}{x_{3}}, \frac{A}{x_{2}}, x_{1}\right\}=\max \left\{\frac{A}{x_{0}}, \frac{A}{x_{-1}}, x_{-2}\right\}=x_{-2}, \\
& x_{5}=\max \left\{\frac{A}{x_{4}}, \frac{A}{x_{3}}, x_{2}\right\}=\max \left\{\frac{A}{x_{-2}}, \frac{A}{x_{0}}, x_{-1}\right\}=x_{-1} .
\end{aligned}
$$

Hence $x_{3}=x_{0}, x_{4}=x_{1}, x_{5}=x_{2}$. From this and by Lemma 2.1 we have that the solution is eventually periodic with period three. Moreover, we have

$$
x_{3 n}=x_{0}, x_{3 n+1}=x_{-2}, x_{3 n+2}=x_{-1}, \quad n \in N_{0},
$$

and we can find the solution of Eq. (1.1) has the following form

$$
\left\{x_{-2}, x_{-1}, x_{0}, x_{-2}, x_{-1}, x_{0}, x_{-2}, x_{-1}, \cdots\right\} \text {. }
$$

Case 3. Assume that $\frac{A}{x_{-1}} \geqslant x_{-2}$ and $\frac{A}{x_{-1}} \geqslant \frac{A}{x_{0}}$, then $x_{1}=\frac{A}{x_{-1}}, x_{0} \geqslant x_{-1}$, and we have

$$
x_{2}=\max \left\{\frac{A}{x_{1}}, \frac{A}{x_{0}}, x_{-1}\right\}=\max \left\{x_{-1}, \frac{A}{x_{0}}, x_{-1}\right\}
$$

(c $c_{1}$ Assume that $x_{-1} \geqslant \frac{A}{x_{0}}$, then $x_{2}=x_{-1}, x_{0} \geqslant \frac{A}{x_{-1}}$, and we have

$$
x_{3}=\max \left\{\frac{A}{x_{2}}, \frac{A}{x_{1}}, x_{0}\right\}=\max \left\{\frac{A}{x_{-1}}, x_{-1}, x_{0}\right\}=x_{0},
$$




$$
\begin{aligned}
& x_{4}=\max \left\{\frac{A}{x_{3}}, \frac{A}{x_{2}}, x_{1}\right\}=\max \left\{\frac{A}{x_{0}}, \frac{A}{x_{-1}}, \frac{A}{x_{-1}}\right\}=\frac{A}{x_{-1}}, \\
& x_{5}=\max \left\{\frac{A}{x_{4}}, \frac{A}{x_{3}}, x_{2}\right\}=\max \left\{x_{-1}, \frac{A}{x_{0}}, x_{-1}\right\}=x_{-1} .
\end{aligned}
$$

Hence $x_{3}=x_{0}, x_{4}=x_{1}, x_{5}=x_{2}$. From this and by Lemma 2.1 we have that the solution is eventually periodic with period three. Moreover, we have

$$
x_{3 n}=x_{0}, x_{3 n+1}=\frac{A}{x_{-1}}, x_{3 n+2}=x_{-1}, \quad n \in N_{0},
$$

and we can find the solution of Eq. (1.1) has the following form

$$
\left\{x_{-2}, x_{-1}, x_{0}, \frac{A}{x_{-1}}, x_{-1}, x_{0}, \frac{A}{x_{-1}}, x_{-1}, \cdots\right\} .
$$

(c) Assume that $\frac{A}{x_{0}} \geqslant x_{-1}$, then $x_{2}=\frac{A}{x_{0}}, \frac{A}{x_{-1}} \geqslant x_{0}$, and we have

$$
\begin{aligned}
& x_{3}=\max \left\{\frac{A}{x_{2}}, \frac{A}{x_{1}}, x_{0}\right\}=\max \left\{x_{0}, x_{-1}, x_{0}\right\}=x_{0}, \\
& x_{4}=\max \left\{\frac{A}{x_{3}}, \frac{A}{x_{2}}, x_{1}\right\}=\max \left\{\frac{A}{x_{0}}, x_{0}, \frac{A}{x_{-1}}\right\}=\frac{A}{x_{-1}}, \\
& x_{5}=\max \left\{\frac{A}{x_{4}}, \frac{A}{x_{3}}, x_{2}\right\}=\max \left\{x_{-1}, \frac{A}{x_{0}}, \frac{A}{x_{0}}\right\}=\frac{A}{x_{0}} .
\end{aligned}
$$

Hence $x_{3}=x_{0}, x_{4}=x_{1}, x_{5}=x_{2}$. From this and by Lemma 2.1 we have that the solution is eventually periodic with period three. Moreover, we have

$$
x_{3 n}=x_{0}, x_{3 n+1}=\frac{A}{x_{-1}}, x_{3 n+2}=\frac{A}{x_{0}}, \quad n \in N_{0},
$$

and we can find the solution of Eq. (1.1) has the following form

$$
\left\{x_{-2}, x_{-1}, x_{0}, \frac{A}{x_{-1}}, \frac{A}{x_{0}}, x_{0}, \frac{A}{x_{-1}}, \frac{A}{x_{0}}, \cdots\right\} .
$$

The proof is completed.

Theorem 2.4. Assume A $>0$ and that initial conditions of Eq. (1.1) satisfy one of conditions (ii)-(vii) in Lemma 2.2. Then every such solution of Eq. (1.1) is eventually periodic with period three.

Proof. If initial conditions of Eq. (1.1) satisfy one of conditions (ii)-(vii) in Lemma 2.2, then by Lemma 2.2, we can obtain that the corresponding solution of Eq. (1.1) is eventually positive. This means that there is $N \in N_{0} \cup\{-2,-1\}$ such that $x_{n}>0$ for every $n \geqslant N$. In particular, we have that $x_{N}, x_{N+1}, x_{N+2}>0$. Since Eq. (1.1) is autonomous if $\left\{x_{n}\right\}_{n=-2}^{\infty}$ is a solution of Eq. (1.1), then $y_{n}=x_{n+N+2}$ is also a solution of Eq. (1.1), but such that $y_{-2}, y_{-1}, y_{0}>0$. Hence the problem is reduced to the case when all the initial conditions are positive. Applying Theorem 2.3, the result follows.

In the next section, we will study the solutions of Eq. (1.1) with the condition $x_{-2}, x_{-1}, x_{0}<0$.

Theorem 2.5. Assume $\mathrm{A}>0$ and $x_{-2}, x_{-1}, x_{0}<0$. Then every solution of $E q$. (1.1) is eventually periodic with period three.

Proof. Since $x_{-2}, x_{-1}, x_{0}<0$ and $A>0$, by the induction and iterative method, we can obtain that $x_{n}<0$ for every $n \in N$. If we use the change $y_{n}=-x_{n}$, then Eq. (1.1) becomes

$$
y_{n+1}=\min \left\{\frac{A}{y_{n}}, \frac{A}{y_{n-1}}, y_{n-2}\right\}
$$

where $y_{n}>0$ for every $n=-2,-1,0, \cdots$. 
Now in order to prove the theorem, we can prove that such solutions of Eq. (2.3) are eventually periodic with period three, from which the result follows. We have

$$
y_{1}=\min \left\{\frac{A}{y_{0}}, \frac{A}{y_{-1}}, y_{-2}\right\}
$$

There are three cases to be considered.

Case 1. Assume that $\frac{A}{y_{0}} \leqslant \frac{A}{y_{-1}}$ and $\frac{A}{y_{0}} \leqslant y_{-2}$, then $y_{1}=\frac{A}{y_{0}}, y_{-1} \leqslant y_{0}, \frac{A}{y_{-2}} \leqslant y_{0}$, and we have

$$
y_{2}=\min \left\{\frac{A}{y_{1}}, \frac{A}{y_{0}}, y_{-1}\right\}=\min \left\{y_{0}, \frac{A}{y_{0}}, y_{-1}\right\}
$$

$\left(a_{1}\right)$ Assume that $\frac{A}{y_{0}} \leqslant y_{-1} \leqslant y_{0}$, then $y_{2}=\frac{A}{y_{0}}$, and we have

$$
\begin{aligned}
& y_{3}=\min \left\{\frac{A}{y_{2}}, \frac{A}{y_{1}}, y_{0}\right\}=\min \left\{y_{0}, y_{0}, y_{0}\right\}=y_{0} \\
& y_{4}=\min \left\{\frac{A}{y_{3}}, \frac{A}{y_{2}}, y_{1}\right\}=\min \left\{\frac{A}{y_{0}}, y_{0}, \frac{A}{y_{0}}\right\}=\frac{A}{y_{0}}, \\
& y_{5}=\min \left\{\frac{A}{y_{4}}, \frac{A}{y_{3}}, y_{2}\right\}=\min \left\{y_{0}, \frac{A}{y_{0}}, \frac{A}{y_{0}}\right\}=\frac{A}{y_{0}} .
\end{aligned}
$$

Hence $y_{3}=y_{0}, y_{4}=y_{1}, y_{5}=y_{2}$. From this and by Lemma 2.1 we have that the solution is eventually periodic with period three. Moreover, we have

$$
y_{3 n}=y_{0}, y_{3 n+1}=\frac{A}{y_{0}}, y_{3 n+2}=\frac{A}{y_{0}}, \quad n \in N_{0},
$$

and we can find the solution of Eq. (1.1) has the following form

$$
\left\{y_{-2}, y_{-1}, y_{0}, \frac{A}{y_{0}}, \frac{A}{y_{0}}, y_{0}, \frac{A}{y_{0}}, \frac{A}{y_{0}}, \cdots\right\}
$$

$\left(a_{2}\right)$ Assume that $y_{-1} \leqslant \frac{A}{y_{0}} \leqslant y_{0}$, then $y_{2}=y_{-1}, y_{0} \leqslant \frac{A}{y_{-1}}$ and we have

$$
\begin{aligned}
& y_{3}=\min \left\{\frac{A}{y_{2}}, \frac{A}{y_{1}}, y_{0}\right\}=\min \left\{\frac{A}{y_{-1}}, y_{0}, y_{0}\right\}=y_{0}, \\
& y_{4}=\min \left\{\frac{A}{y_{3}}, \frac{A}{y_{2}}, y_{1}\right\}=\min \left\{\frac{A}{y_{0}}, \frac{A}{y_{-1}}, \frac{A}{y_{0}}\right\}=\frac{A}{y_{0}}, \\
& y_{5}=\min \left\{\frac{A}{y_{4}}, \frac{A}{y_{3}}, y_{2}\right\}=\min \left\{y_{0}, \frac{A}{y_{0}}, y_{-1}\right\}=y_{-1} .
\end{aligned}
$$

Hence $y_{3}=y_{0}, y_{4}=y_{1}, y_{5}=y_{2}$. From this and by Lemma 2.1 we have that the solution is eventually periodic with period three. Moreover, we have

$$
y_{3 n}=y_{0}, y_{3 n+1}=\frac{A}{y_{0}}, y_{3 n+2}=y_{-1}, \quad n \in N_{0},
$$

and we can find the solution of Eq. (1.1) has the following form

$$
\left\{y_{-2}, y_{-1}, y_{0}, \frac{A}{y_{0}}, y_{-1}, y_{0}, \frac{A}{y_{0}}, y_{-1}, \cdots\right\}
$$

$\left(a_{3}\right)$ Assume that $y_{-1} \leqslant y_{0} \leqslant \frac{A}{y_{0}}$, then $y_{2}=y_{-1}, y_{0} \leqslant \frac{A}{y_{-1}}$ and we have

$$
y_{3}=\min \left\{\frac{A}{y_{2}}, \frac{A}{y_{1}}, y_{0}\right\}=\min \left\{\frac{A}{y_{-1}}, y_{0}, y_{0}\right\}=y_{0},
$$




$$
\begin{aligned}
& y_{4}=\min \left\{\frac{A}{y_{3}}, \frac{A}{y_{2}}, y_{1}\right\}=\min \left\{\frac{A}{y_{0}}, \frac{A}{y_{-1}}, \frac{A}{y_{0}}\right\}=\frac{A}{y_{0}}, \\
& y_{5}=\min \left\{\frac{A}{y_{4}}, \frac{A}{y_{3}}, y_{2}\right\}=\min \left\{y_{0}, \frac{A}{y_{0}}, y_{-1}\right\}=y_{-1} .
\end{aligned}
$$

Hence $y_{3}=y_{0}, y_{4}=y_{1}, y_{5}=y_{2}$. From this and by Lemma 2.1 we have that the solution is eventually periodic with period three. Moreover, we have

$$
y_{3 n}=y_{0}, y_{3 n+1}=\frac{A}{y_{0}}, y_{3 n+2}=y_{-1}, \quad n \in N_{0},
$$

and we can find the solution of Eq. (1.1) has the following form

$$
\left\{y_{-2}, y_{-1}, y_{0}, \frac{A}{y_{0}}, y_{-1}, y_{0}, \frac{A}{y_{0}}, y_{-1}, \cdots\right\}
$$

Case 2. Assume that $y_{-2} \leqslant \frac{A}{y_{0}}$ and $y_{-2} \leqslant \frac{A}{y_{-1}}$, then $y_{1}=y_{-2}, y_{-1} \leqslant \frac{A}{y_{-2}}, y_{0} \leqslant \frac{A}{y_{-2}}$, and we have

$$
y_{2}=\min \left\{\frac{A}{y_{1}}, \frac{A}{y_{0}}, y_{-1}\right\}=\min \left\{\frac{A}{y_{-2}}, \frac{A}{y_{0}}, y_{-1}\right\}
$$

$\left(b_{1}\right)$ Assume that $\frac{A}{y_{0}} \leqslant y_{-1} \leqslant \frac{A}{y_{-2}}$, then $y_{2}=\frac{A}{y_{0}}, y_{-2} \leqslant y_{0}$, and we have

$$
\begin{aligned}
& y_{3}=\min \left\{\frac{A}{y_{2}}, \frac{A}{y_{1}}, y_{0}\right\}=\min \left\{y_{0}, \frac{A}{y_{-2}}, y_{0}\right\}=y_{0}, \\
& y_{4}=\min \left\{\frac{A}{y_{3}}, \frac{A}{y_{2}}, y_{1}\right\}=\min \left\{\frac{A}{y_{0}}, y_{0}, y_{-2}\right\}=y_{-2}, \\
& y_{5}=\min \left\{\frac{A}{y_{4}}, \frac{A}{y_{3}}, y_{2}\right\}=\min \left\{\frac{A}{y_{-2}}, \frac{A}{y_{0}}, \frac{A}{y_{0}}\right\}=\frac{A}{y_{0}} .
\end{aligned}
$$

Hence $y_{3}=y_{0}, y_{4}=y_{1}, y_{5}=y_{2}$. From this and by Lemma 2.1 we have that the solution is eventually periodic with period three. Moreover, we have

$$
y_{3 n}=y_{0}, y_{3 n+1}=y_{-2}, y_{3 n+2}=\frac{A}{y_{0}}, \quad n \in N_{0},
$$

and we can find the solution of Eq. (1.1) has the following form

$$
\left\{y_{-2}, y_{-1}, y_{0}, y_{-2}, \frac{A}{y_{0}}, y_{0}, y_{-2}, \frac{A}{y_{0}}, \cdots\right\} \text {. }
$$

$\left(b_{2}\right)$ Assume that $y_{-1} \leqslant \frac{A}{y_{0}} \leqslant \frac{A}{y_{-2}}$, then $y_{2}=y_{-1}, y_{0} \leqslant \frac{A}{y_{-1}}$, and we have

$$
\begin{aligned}
& y_{3}=\min \left\{\frac{A}{y_{2}}, \frac{A}{y_{1}}, y_{0}\right\}=\min \left\{\frac{A}{y_{-1}}, \frac{A}{y_{-2}}, y_{0}\right\}=y_{0}, \\
& y_{4}=\min \left\{\frac{A}{y_{3}}, \frac{A}{y_{2}}, y_{1}\right\}=\min \left\{\frac{A}{y_{0}}, \frac{A}{y_{-1}}, y_{-2}\right\}=y_{-2}, \\
& y_{5}=\min \left\{\frac{A}{y_{4}}, \frac{A}{y_{3}}, y_{2}\right\}=\min \left\{\frac{A}{y_{-2}}, \frac{A}{y_{0}}, y_{-1}\right\}=y_{-1} .
\end{aligned}
$$

Hence $y_{3}=y_{0}, y_{4}=y_{1}, y_{5}=y_{2}$. From this and by Lemma 2.1 we have that the solution is eventually periodic with period three. Moreover, we have

$$
y_{3 n}=y_{0}, y_{3 n+1}=y_{-2}, y_{3 n+2}=y_{-1}, \quad n \in N_{0},
$$

and we can find the solution of Eq. (1.1) has the following form

$$
\left\{y_{-2}, y_{-1}, y_{0}, y_{-2}, x_{-1}, y_{0}, y_{-2}, y_{-1}, \cdots\right\}
$$


$\left(b_{3}\right)$ Assume that $y_{-1} \leqslant \frac{A}{y_{-2}} \leqslant \frac{A}{y_{0}}$, then $y_{2}=y_{-1}, y_{0} \leqslant \frac{A}{y_{-1}}$, and we have

$$
\begin{aligned}
& y_{3}=\min \left\{\frac{A}{y_{2}}, \frac{A}{y_{1}}, y_{0}\right\}=\min \left\{\frac{A}{y_{-1}}, \frac{A}{y_{-2}}, y_{0}\right\}=y_{0} \\
& y_{4}=\min \left\{\frac{A}{y_{3}}, \frac{A}{y_{2}}, y_{1}\right\}=\min \left\{\frac{A}{y_{0}}, \frac{A}{y_{-1}}, y_{-2}\right\}=y_{-2} \\
& y_{5}=\min \left\{\frac{A}{y_{4}}, \frac{A}{y_{3}}, y_{2}\right\}=\min \left\{\frac{A}{y_{-2}}, \frac{A}{y_{0}}, y_{-1}\right\}=y_{-1}
\end{aligned}
$$

Hence $y_{3}=y_{0}, y_{4}=y_{1}, y_{5}=y_{2}$. From this and by Lemma 2.1 we have that the solution is eventually periodic with period three. Moreover, we have

$$
y_{3 n}=y_{0}, y_{3 n+1}=y_{-2}, y_{3 n+2}=y_{-1}, \quad n \in N_{0},
$$

and we can find the solution of Eq. (1.1) has the following form

$$
\left\{y_{-2}, y_{-1}, y_{0}, y_{-2}, y_{-1}, y_{0}, y_{-2}, y_{-1}, \cdots\right\} \text {. }
$$

Case 3. Assume that $\frac{A}{y_{-1}} \leqslant y_{-2}$ and $\frac{A}{y_{-1}} \leqslant \frac{A}{y_{0}}$, then $y_{1}=\frac{A}{y_{-1}}, y_{0} \leqslant y_{-1}$, and we have

$$
y_{2}=\min \left\{\frac{A}{y_{1}}, \frac{A}{y_{0}}, y_{-1}\right\}=\min \left\{y_{-1}, \frac{A}{y_{0}}, y_{-1}\right\}
$$

(c 1 ) Assume that $y_{-1} \leqslant \frac{A}{y_{0}}$, then $y_{2}=y_{-1}, y_{0} \leqslant \frac{A}{y_{-1}}$, and we have

$$
\begin{aligned}
& y_{3}=\min \left\{\frac{A}{y_{2}}, \frac{A}{y_{1}}, y_{0}\right\}=\min \left\{\frac{A}{y_{-1}}, y_{-1}, y_{0}\right\}=y_{0}, \\
& y_{4}=\min \left\{\frac{A}{y_{3}}, \frac{A}{y_{2}}, y_{1}\right\}=\min \left\{\frac{A}{y_{0}}, \frac{A}{y_{-1}}, \frac{A}{y_{-1}}\right\}=\frac{A}{y_{-1}}, \\
& y_{5}=\min \left\{\frac{A}{y_{4}}, \frac{A}{y_{3}}, y_{2}\right\}=\min \left\{y_{-1}, \frac{A}{y_{0}}, y_{-1}\right\}=y_{-1} .
\end{aligned}
$$

Hence $y_{3}=y_{0}, y_{4}=y_{1}, y_{5}=y_{2}$. From this and by Lemma 2.1 we have that the solution is eventually periodic with period three. Moreover, we have

$$
y_{3 n}=y_{0}, y_{3 n+1}=\frac{A}{y_{-1}}, y_{3 n+2}=y_{-1}, \quad n \in N_{0},
$$

and we can find the solution of Eq. (1.1) has the following form

$$
\left\{y_{-2}, y_{-1}, y_{0}, \frac{A}{y_{-1}}, y_{-1}, y_{0}, \frac{A}{y_{-1}}, y_{-1}, \cdots\right\}
$$

(c) Assume that $\frac{A}{y_{0}} \leqslant y_{-1}$, then $y_{2}=\frac{A}{y_{0}}, \frac{A}{y_{-1}} \leqslant y_{0}$, and we have

$$
\begin{aligned}
& y_{3}=\min \left\{\frac{A}{y_{2}}, \frac{A}{y_{1}}, y_{0}\right\}=\min \left\{y_{0}, y_{-1}, y_{0}\right\}=y_{0}, \\
& y_{4}=\min \left\{\frac{A}{y_{3}}, \frac{A}{y_{2}}, y_{1}\right\}=\min \left\{\frac{A}{y_{0}}, y_{0}, \frac{A}{y_{-1}}\right\}=\frac{A}{y_{-1}}, \\
& y_{5}=\min \left\{\frac{A}{y_{4}}, \frac{A}{y_{3}}, y_{2}\right\}=\min \left\{y_{-1}, \frac{A}{y_{0}}, \frac{A}{y_{0}}\right\}=\frac{A}{y_{0}} .
\end{aligned}
$$

Hence $y_{3}=y_{0}, y_{4}=y_{1}, y_{5}=y_{2}$. From this and by Lemma 2.1 we have that the solution is eventually 
periodic with period three. Moreover, we have

$$
y_{3 n}=y_{0}, y_{3 n+1}=\frac{A}{y_{-1}}, y_{3 n+2}=\frac{A}{y_{0}}, \quad n \in N_{0},
$$

and we can find the solution of Eq. (1.1) has the following form

$$
\left\{y_{-2}, y_{-1}, y_{0}, \frac{A}{y_{-1}}, \frac{A}{y_{0}}, y_{0}, \frac{A}{y_{-1}}, \frac{A}{y_{0}}, \cdots\right\}
$$

The proof is completed.

\subsection{The case $\mathrm{A}<0$.}

In this section we study the solutions of (1.1) when $A<0$.

Theorem 2.6. Assume that $\mathrm{A}<0$. Then every solution of Eq. (1.1) is eventually periodic with period three.

Proof. There are eight cases to be considered.

Case 1. $x_{-2}, x_{-1}, x_{0}>0$. Since $A<0$ we have

$$
x_{1}=\max \left\{\frac{A}{x_{0}}, \frac{A}{x_{-1}}, x_{-2}\right\} .
$$

From this, (1.1) and by induction we have $x_{n}>0$ for every $n \in N_{0}$. Hence

$$
x_{n+1}=\max \left\{\frac{A}{x_{n}}, \frac{A}{x_{n-1}}, x_{n-2}\right\}=x_{n-2},
$$

from which the result follows in this case.

Case 2. $x_{-2}, x_{0}>0, x_{-1}<0$. Since $A<0$ we have

$$
x_{1}=\max \left\{\frac{A}{x_{0}}, \frac{A}{x_{-1}}, x_{-2}\right\}=\max \left\{\frac{A}{x_{-1}}, x_{-2}\right\}>0 .
$$

$\left(a_{1}\right)$ If $\frac{A}{x_{-1}} \geqslant x_{-2}$, then $x_{1}=\frac{A}{x_{-1}}, x_{-1} \geqslant \frac{A}{x_{-2}}$. Further we have

$$
x_{2}=\max \left\{\frac{A}{x_{1}}, \frac{A}{x_{0}}, x_{-1}\right\}=\max \left\{x_{-1}, \frac{A}{x_{0}}, x_{-1}\right\}<0 .
$$

There exist two subcases.

$\left(a_{11}\right)$ If $x_{-1} \geqslant \frac{A}{x_{0}}$, then $x_{2}=x_{-1}, x_{0} \leqslant \frac{A}{x_{-1}}$, we have

$$
\begin{aligned}
& x_{3}=\max \left\{\frac{A}{x_{2}}, \frac{A}{x_{1}}, x_{0}\right\}=\max \left\{\frac{A}{x_{-1}}, x_{-1}, x_{0}\right\}=\frac{A}{x_{-1}}>0, \\
& x_{4}=\max \left\{\frac{A}{x_{3}}, \frac{A}{x_{2}}, x_{1}\right\}=\max \left\{x_{-1}, \frac{A}{x_{-1}}, \frac{A}{x_{-1}}\right\}=\frac{A}{x_{-1}}>0, \\
& x_{5}=\max \left\{\frac{A}{x_{4}}, \frac{A}{x_{3}}, x_{2}\right\}=\max \left\{x_{-1}, x_{-1}, x_{-1}\right\}=x_{-1}<0, \\
& x_{6}=\max \left\{\frac{A}{x_{5}}, \frac{A}{x_{4}}, x_{3}\right\}=\max \left\{\frac{A}{x_{-1}}, x_{-1}, \frac{A}{x_{-1}}\right\}=\frac{A}{x_{-1}}>0, \\
& x_{7}=\max \left\{\frac{A}{x_{6}}, \frac{A}{x_{5}}, x_{4}\right\}=\max \left\{x_{-1}, \frac{A}{x_{-1}}, \frac{A}{x_{-1}}\right\}=\frac{A}{x_{-1}}>0 .
\end{aligned}
$$


Hence $x_{5}=x_{2}, x_{6}=x_{3}, x_{7}=x_{4}$, from which the result follows in the case. $\left(a_{12}\right)$ If $\frac{A}{x_{0}} \geqslant x_{-1}$, then $x_{2}=\frac{A}{x_{0}}, x_{0} \geqslant \frac{A}{x_{-1}}$, we have

$$
\begin{aligned}
& x_{3}=\max \left\{\frac{A}{x_{2}}, \frac{A}{x_{1}}, x_{0}\right\}=\max \left\{x_{0}, x_{-1}, x_{0}\right\}=x_{0}>0 \\
& x_{4}=\max \left\{\frac{A}{x_{3}}, \frac{A}{x_{2}}, x_{1}\right\}=\max \left\{\frac{A}{x_{0}}, x_{0}, \frac{A}{x_{-1}}\right\}=x_{0}>0, \\
& x_{5}=\max \left\{\frac{A}{x_{4}}, \frac{A}{x_{3}}, x_{2}\right\}=\max \left\{\frac{A}{x_{0}}, \frac{A}{x_{0}}, \frac{A}{x_{0}}\right\}=\frac{A}{x_{0}}<0, \\
& x_{6}=\max \left\{\frac{A}{x_{5}}, \frac{A}{x_{4}}, x_{3}\right\}=\max \left\{x_{0}, \frac{A}{x_{0}}, x_{0}\right\}=x_{0}>0 \\
& x_{7}=\max \left\{\frac{A}{x_{6}}, \frac{A}{x_{5}}, x_{4}\right\}=\max \left\{\frac{A}{x_{0}}, x_{0}, x_{0}\right\}=x_{0}>0
\end{aligned}
$$

Hence $x_{5}=x_{2}, x_{6}=x_{3}, x_{7}=x_{4}$, from which the result follows in the case.

$\left(a_{2}\right)$ If $x_{-2} \geqslant \frac{A}{x_{-1}}$, then $x_{1}=x_{-2}, \frac{A}{x_{-2}} \geqslant x_{-1}$, Further we have

$$
x_{2}=\max \left\{\frac{A}{x_{1}}, \frac{A}{x_{0}}, x_{-1}\right\}=\max \left\{\frac{A}{x_{-2}}, \frac{A}{x_{0}}, x_{-1}\right\}<0 .
$$

Now we have three subcases.

$\left(a_{21}\right)$ If $\frac{A}{x_{0}} \geqslant \frac{A}{x_{-2}} \geqslant x_{-1}$, then $x_{2}=\frac{A}{x_{0}}, x_{0} \geqslant x_{-2}$, then we have

$$
\begin{aligned}
& x_{3}=\max \left\{\frac{A}{x_{2}}, \frac{A}{x_{1}}, x_{0}\right\}=\max \left\{x_{0}, \frac{A}{x_{-2}}, x_{0}\right\}=x_{0}>0, \\
& x_{4}=\max \left\{\frac{A}{x_{3}}, \frac{A}{x_{2}}, x_{1}\right\}=\max \left\{\frac{A}{x_{0}}, x_{0}, x_{-2}\right\}=x_{0}>0, \\
& x_{5}=\max \left\{\frac{A}{x_{4}}, \frac{A}{x_{3}}, x_{2}\right\}=\max \left\{\frac{A}{x_{0}}, \frac{A}{x_{0}}, \frac{A}{x_{0}}\right\}=\frac{A}{x_{0}}<0, \\
& x_{6}=\max \left\{\frac{A}{x_{5}}, \frac{A}{x_{4}}, x_{3}\right\}=\max \left\{x_{0}, \frac{A}{x_{0}}, x_{0}\right\}=x_{0}>0 \\
& x_{7}=\max \left\{\frac{A}{x_{6}}, \frac{A}{x_{5}}, x_{4}\right\}=\max \left\{\frac{A}{x_{0}}, x_{0}, x_{0}\right\}=x_{0}>0 .
\end{aligned}
$$

Hence $x_{5}=x_{2}, x_{6}=x_{3}, x_{7}=x_{4}$, from which the result follows in the case. $\left(a_{22}\right)$ If $\frac{A}{x_{-2}} \geqslant \frac{A}{x_{0}} \geqslant x_{-1}$, then $x_{2}=\frac{A}{x_{-2}}, x_{-2} \geqslant x_{0}$, then we have

$$
\begin{aligned}
& x_{3}=\max \left\{\frac{A}{x_{2}}, \frac{A}{x_{1}}, x_{0}\right\}=\max \left\{x_{-2}, \frac{A}{x_{-2}}, x_{0}\right\}=x_{-2}>0, \\
& x_{4}=\max \left\{\frac{A}{x_{3}}, \frac{A}{x_{2}}, x_{1}\right\}=\max \left\{\frac{A}{x_{-2}}, x_{-2}, x_{-2}\right\}=x_{-2}>0 \\
& x_{5}=\max \left\{\frac{A}{x_{4}}, \frac{A}{x_{3}}, x_{2}\right\}=\max \left\{\frac{A}{x_{-2}}, \frac{A}{x_{-2}}, \frac{A}{x_{-2}}\right\}=\frac{A}{x_{-2}}<0, \\
& x_{6}=\max \left\{\frac{A}{x_{5}}, \frac{A}{x_{4}}, x_{3}\right\}=\max \left\{x_{-2}, \frac{A}{x_{-2}}, x_{-2}\right\}=x_{-2}>0 \\
& x_{7}=\max \left\{\frac{A}{x_{6}}, \frac{A}{x_{5}}, x_{4}\right\}=\max \left\{\frac{A}{x_{-2}}, x_{-2}, x_{-2}\right\}=x_{-2}>0 .
\end{aligned}
$$

Hence $x_{5}=x_{2}, x_{6}=x_{3}, x_{7}=x_{4}$, from which the result follows in the case. 
$\left(a_{23}\right)$ If $\frac{A}{x_{-2}} \geqslant x_{-1} \geqslant \frac{A}{x_{0}}$, then $x_{2}=\frac{A}{x_{-2}}, x_{-2} \geqslant x_{0}$, then we have

$$
\begin{aligned}
& x_{3}=\max \left\{\frac{A}{x_{2}}, \frac{A}{x_{1}}, x_{0}\right\}=\max \left\{x_{-2}, \frac{A}{x_{-2}}, x_{0}\right\}=x_{-2}>0, \\
& x_{4}=\max \left\{\frac{A}{x_{3}}, \frac{A}{x_{2}}, x_{1}\right\}=\max \left\{\frac{A}{x_{-2}}, x_{-2}, x_{-2}\right\}=x_{-2}>0, \\
& x_{5}=\max \left\{\frac{A}{x_{4}}, \frac{A}{x_{3}}, x_{2}\right\}=\max \left\{\frac{A}{x_{-2}}, \frac{A}{x_{-2}}, \frac{A}{x_{-2}}\right\}=\frac{A}{x_{-2}}<0, \\
& x_{6}=\max \left\{\frac{A}{x_{5}}, \frac{A}{x_{4}}, x_{3}\right\}=\max \left\{x_{-2}, \frac{A}{x_{-2}}, x_{-2}\right\}=x_{-2}>0, \\
& x_{7}=\max \left\{\frac{A}{x_{6}}, \frac{A}{x_{5}}, x_{4}\right\}=\max \left\{\frac{A}{x_{-2}}, x_{-2}, x_{-2}\right\}=x_{-2}>0 .
\end{aligned}
$$

Hence $x_{5}=x_{2}, x_{6}=x_{3}, x_{7}=x_{4}$, from which the result follows in the case.

Case 3. $x_{0}>0, x_{-2}, x_{-1}<0$, since $A<0$, we have

$$
x_{1}=\max \left\{\frac{A}{x_{0}}, \frac{A}{x_{-1}}, x_{-2}\right\}=\frac{A}{x_{-1}}>0, \quad x_{2}=\max \left\{\frac{A}{x_{1}}, \frac{A}{x_{0}}, x_{-1}\right\}=\max \left\{x_{-1}, \frac{A}{x_{0}}, x_{-1}\right\} .
$$

Now we have two cases.

$\left(b_{1}\right)$ If $x_{-1} \geqslant \frac{A}{x_{0}}$, then we have $x_{2}=x_{-1}, x_{0} \leqslant \frac{A}{x_{-1}}$. Further we have

$$
\begin{aligned}
& x_{3}=\max \left\{\frac{A}{x_{2}}, \frac{A}{x_{1}}, x_{0}\right\}=\max \left\{\frac{A}{x_{-1}}, x_{-1}, x_{0}\right\}=\frac{A}{x_{-1}}>0, \\
& x_{4}=\max \left\{\frac{A}{x_{3}}, \frac{A}{x_{2}}, x_{1}\right\}=\max \left\{x_{-1}, \frac{A}{x_{-1}}, \frac{A}{x_{-1}}\right\}=\frac{A}{x_{-1}}>0, \\
& x_{5}=\max \left\{\frac{A}{x_{4}}, \frac{A}{x_{3}}, x_{2}\right\}=\max \left\{x_{-1}, x_{-1}, x_{-1}\right\}=x_{-1}<0 \\
& x_{6}=\max \left\{\frac{A}{x_{5}}, \frac{A}{x_{4}}, x_{3}\right\}=\max \left\{\frac{A}{x_{-1}}, x_{-1}, \frac{A}{x_{-1}}\right\}=\frac{A}{x_{-1}}>0 .
\end{aligned}
$$

Hence $x_{4}=x_{1}, x_{5}=x_{2}, x_{6}=x_{3}$, from which the result follows in the case. $\left(b_{2}\right)$ If $\frac{A}{x_{0}} \geqslant x_{-1}$, then we have $x_{2}=\frac{A}{x_{0}}, x_{0} \geqslant \frac{A}{x_{-1}}$. Further we have

$$
\begin{aligned}
& x_{3}=\max \left\{\frac{A}{x_{2}}, \frac{A}{x_{1}}, x_{0}\right\}=\max \left\{x_{0}, x_{-1}, x_{0}\right\}=x_{0}>0, \\
& x_{4}=\max \left\{\frac{A}{x_{3}}, \frac{A}{x_{2}}, x_{1}\right\}=\max \left\{\frac{A}{x_{0}}, x_{0}, \frac{A}{x_{-1}}\right\}=x_{0}>0, \\
& x_{5}=\max \left\{\frac{A}{x_{4}}, \frac{A}{x_{3}}, x_{2}\right\}=\max \left\{\frac{A}{x_{0}}, \frac{A}{x_{0}}, \frac{A}{x_{0}}\right\}=\frac{A}{x_{0}}<0, \\
& x_{6}=\max \left\{\frac{A}{x_{5}}, \frac{A}{x_{4}}, x_{3}\right\}=\max \left\{x_{0}, \frac{A}{x_{0}}, x_{0}\right\}=x_{0}>0, \\
& x_{7}=\max \left\{\frac{A}{x_{6}}, \frac{A}{x_{5}}, x_{4}\right\}=\max \left\{\frac{A}{x_{0}}, x_{0}, x_{0}\right\}=x_{0}>0 .
\end{aligned}
$$

Hence $x_{5}=x_{2}, x_{6}=x_{3}, x_{7}=x_{4}$, from which the result follows in the case.

Case 4. $x_{-2}, x_{-1}, x_{0}<0$. Since $A<0$, we have

$$
x_{1}=\max \left\{\frac{A}{x_{0}}, \frac{A}{x_{-1}}, x_{-2}\right\}=\max \left\{\frac{A}{x_{0}}, \frac{A}{x_{-1}}\right\}>0 .
$$

Now we have two cases. 
(c 1 ) If $\frac{A}{x_{0}} \geqslant \frac{A}{x_{-1}}$, then $x_{1}=\frac{A}{x_{0}}$. Further we have

$$
\begin{aligned}
& x_{2}=\max \left\{\frac{A}{x_{1}}, \frac{A}{x_{0}}, x_{-1}\right\}=\max \left\{x_{0}, \frac{A}{x_{0}}, x_{-1}\right\}=\frac{A}{x_{0}}>0 \\
& x_{3}=\max \left\{\frac{A}{x_{2}}, \frac{A}{x_{1}}, x_{0}\right\}=\max \left\{x_{0}, x_{0}, x_{0}\right\}=x_{0}<0 \\
& x_{4}=\max \left\{\frac{A}{x_{3}}, \frac{A}{x_{2}}, x_{1}\right\}=\max \left\{\frac{A}{x_{0}}, x_{0}, \frac{A}{x_{0}}\right\}=\frac{A}{x_{0}}>0 \\
& x_{5}=\max \left\{\frac{A}{x_{4}}, \frac{A}{x_{3}}, x_{2}\right\}=\max \left\{x_{0}, \frac{A}{x_{0}}, \frac{A}{x_{0}}\right\}=\frac{A}{x_{0}}>0 .
\end{aligned}
$$

Hence $x_{3}=x_{0}, x_{4}=x_{1}, x_{5}=x_{2}$, from which the result follows in the case.

(c $c_{2}$ If $\frac{A}{x_{-1}} \geqslant \frac{A}{x_{0}}$, then $x_{1}=\frac{A}{x_{-1}}, x_{0} \geqslant x_{-1}$. Further we have

$$
\begin{aligned}
& x_{2}=\max \left\{\frac{A}{x_{1}}, \frac{A}{x_{0}}, x_{-1}\right\}=\max \left\{x_{-1}, \frac{A}{x_{0}}, x_{-1}\right\}=\frac{A}{x_{0}}>0, \\
& x_{3}=\max \left\{\frac{A}{x_{2}}, \frac{A}{x_{1}}, x_{0}\right\}=\max \left\{x_{0}, x_{-1}, x_{0}\right\}=x_{0}<0 \\
& x_{4}=\max \left\{\frac{A}{x_{3}}, \frac{A}{x_{2}}, x_{1}\right\}=\max \left\{\frac{A}{x_{0}}, x_{0}, \frac{A}{x_{-1}}\right\}=\frac{A}{x_{-1}}>0 \\
& x_{5}=\max \left\{\frac{A}{x_{4}}, \frac{A}{x_{3}}, x_{2}\right\}=\max \left\{x_{-1}, \frac{A}{x_{0}}, \frac{A}{x_{0}}\right\}=\frac{A}{x_{0}}>0 .
\end{aligned}
$$

Hence $x_{3}=x_{0}, x_{4}=x_{1}, x_{5}=x_{2}$, from which the result follows in the case.

In the same way, when $x_{-2}>0, x_{-1}, x_{0}<0$ or $x_{-1}>0, x_{-2}, x_{0}<0$ or $x_{-2}, x_{-1}>0, x_{0}<0$ or $x_{-1}, x_{0}>0, x_{-2}<0$, we can imply the result in the case. This completes the proof of the theorem.

From Remark 1.1 and Theorems 2.3, 2.4, 2.5, 2.6 the following result follows.

Theorem 2.7. Let $A \in R$, then every well-defined solution of Eq. (1.1) is eventually periodic with period three.

\section{Acknowledgment}

This work is supported Science Fund for Distinguished Young Scholars (cstc2014jcyjjq40004) of China, the National Nature Science Fund (Project nos. 11372366 and 61503053) of China, the Natural Science Foundation Project of CQ CSTC (Grant nos. cstc2015jcyjBX0135 and cstc2015jjA20016) of China, the Science and Technology Project of Chongqing Municipal Education Committee (Grants no. kj1400423) of China, the Postdoctoral Science Foundation (Grant no. 2016m602663) of China, and the Excellent Talents Project of Colleges and Universities in Chongqing of China.

\section{References}

[1] M. M. El-Dessoky, On the periodicity of solutions of max-type difference equation, Math. Methods Appl. Sci., 38 (2015), 3295-3307. 1

[2] E. M. Elsayed, B. Iričanin, S. Stević, On the max-type equation $x_{n+1}=\max \left\{\frac{A_{n}}{x_{n}}, x_{n-1}\right\}$, Ars Combin., 95 (2010), 187-192. 1

[3] E. M. Elsayed, S. Stević, On the max-type equation $x_{n+1}=\max \left\{\frac{A}{x_{n}}, x_{n-2}\right\}$, Nonlinear Anal., 71 (2009), $910-922.1$

[4] A. Gelişken, C. Çinar, On the global attractivity of a max-type difference equation, Discrete Dyn. Nat. Soc., 2009 (2009), 5 pages. 1

[5] T. F. Ibrahim, N. Touafek, Max-type system of difference equations with positive two-periodic sequences, Math. Methods Appl. Sci., 37 (2014), 2541-2553. 1

[6] B. D. Iričanin, E. M. Elsayed, On the max-type difference equation $x_{n+1}=\max \left\{A / x_{n}, x_{n-3}\right\}$, Discrete Dyn. Nat. Soc., 2010 (2010), 13 pages. 1 
[7] W. T. Jamieson, O. Merino, Asymptotic behavior results for solutions to some nonlinear difference equations, J. Math. Anal. Appl., 430 (2015), 614-632. 1

[8] D. Jana, E. M. Elsayed, Interplay between strong Allee effect, harvesting and hydra effect of a single population discrete-time system, Int. J. Biomath., 9 (2016), 25 pages. 1

[9] L.-J. Kong, Homoclinic solutions for a second order difference equation with p-Laplacian, Appl. Math. Comput., 247 (2014), 1113-1121. 1

[10] W.-T. Li, Y.-H. Zhang, Y.-H. Su, Global attractivity in a class of higher-order nonlinear difference equation, Acta Math. Sci. Ser. B Engl. Ed., 25 (2005), 59-66. 1

[11] W.-P. Liu, S. Stević, Global attractivity of a family of nonautonomous max-type difference equations, Appl. Math. Comput., 218 (2012), 6297-6303. 1

[12] J. E. Macías-Díaz, A positive finite-difference model in the computational simulation of complex biological film models, J. Difference Equ. Appl., 20 (2014), 548-569. 1

[13] J. Migda, Approximative solutions to difference equations of neutral type, Appl. Math. Comput., 268 (2015), 763-774. 1

[14] D. P. Mishev, W. T. Patula, H. D. Voulov, A reciprocal difference equation with maximum, Comput. Math. Appl., 43 (2002), 1021-1026. 1

[15] A. D. Myškis, Some problems in the theory of differential equations with deviating argument, (Russian) Uspehi Mat. Nauk, 32 (1977), 173-202. 1

[16] I. Niven, H. S. Zuckerman, H. L. Montgomery, An introduction to the theory of numbers, Fifth edition, John Wiley \& Sons, Inc., New York, (1991). 1

[17] W. T. Patula, H. D. Voulov, On a max type recurrence relation with periodic coefficients, J. Differ. Equ. Appl., 10 (2004), 329-338. 1

[18] E. C. Pielou, Population and community ecology: principles and methods, Gordon and Breach Science Publishers, Inc., New York, (1974). 1

[19] E. P. Popov, Automatic regulation and control, (Russian) Nauka, Moscow, (1966). 1

[20] B. Qin, T.-X. Sun, H.-J. Xi, Dynamics of the max-type difference equation $x_{n+1}=\max \left\{\frac{A}{x_{n}}, x_{n-k}\right\}$, J. Comput. Anal. Appl., 14 (2012), 856-861. 1

[21] M. Shojaei, R. Saadati, H. Adibi, Stability and periodic character of a rational third order difference equation, Chaos Solitons Fractals, 39 (2009), 1203-1209. 1

[22] S. Stević, On a nonlinear generalized max-type difference equation, J. Math. Anal. Appl., 376 (2011), 317-328. 1

[23] S. Stević, On a symmetric system of max-type difference equations, Appl. Math. Comput., 219 (2013), 8407-8412. 1

[24] T.-X. Sun, Q.-L. He, X. Wu, H.-J. Xi, Global behavior of the max-type difference equation $x_{n}=\max \left\{\frac{1}{x_{n-m}}, \frac{A_{n}}{x_{n-r}}\right\}$, Appl. Math. Comput., 248 (2014), 687-692. 1

[25] Q. Xiao, Q.-H. Shi, Eventually periodic solutions of a max-type equation, Math. Comput. Modelling, 57 (2013), $992-996$. 1 